\title{
Retention outcomes and drivers of loss among HIV-exposed and infected infants in Uganda: a retrospective cohort study
}

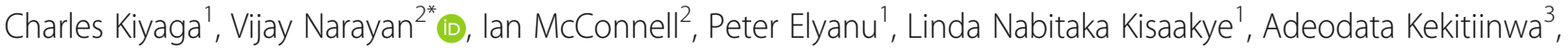
Matthew Price ${ }^{2}$ and Jeff Grosz ${ }^{2}$

\begin{abstract}
Background: Uganda's HIV Early Infant Diagnosis (EID) program rapidly scaled up testing of HIV-exposed infants (HEI) in its early years. However, little was known about retention outcomes of HEl after testing. Provision of transport refunds to HEl caregivers was piloted at 3 hospitals to improve retention. This study was conducted to quantify retention outcomes of tested HEl, identify factors driving loss-to-follow-up, and assess the effect of transport refunds on HEl retention.
\end{abstract}

Methods: This mixed-methods study included 7 health facilities - retrospective cohort review at 3 hospitals and qualitative assessment at all facilities. The cohort comprised all HEl tested from September-2007 to February-2009. Retention data was collected manually at each hospital. Qualitative methods included health worker interviews and structured clinic observation. Qualitative data was synthesized, analyzed and triangulated to identify factors driving HEl loss-to-follow-up.

Results: The cohort included $1268 \mathrm{HEl}$, with 244 testing HIV-positive. Only 57\% (718/1268) of tested HEl received results. The transport refund pilot increased the percent of $\mathrm{HEl}$ caregivers receiving test results from $54 \%(n=763)$ to $58 \%(n=505)(p=.08)$. HEl were tested at late ages (Mean $=7.0$ months, $n=1268)$. Many HEl weren't tested at all: at 1 hospital, only 18\% (67/367) of HIV+ pregnant women brought their HEl for testing after birth. Among HIV+ infants, only 40\% (98/244) received results and enrolled at an ART Clinic. Of enrolled HIV+ infants, only 43\% (57/98) were still active in chronic care. 36\% (27/75) of eligible HIV+ infants started ART. Our analysis identified 6 categories of factors driving HEl loss-to-follow-up: fragmentation of EID services across several clinics, with most poorly equipped for HEl care/follow-up; poor referral mechanisms and data management systems; inconsistent clinical care; substandard counseling; poor health worker knowledge of EID; long sample-result turnaround times.

Discussion: The poor outcomes for HEl and HIV+ infants have highlighted an urgent need to improve retention and linkage to care. To address the identified gaps, Uganda's Ministry of Health and the Clinton Health Access Initiative developed a new implementation model, shifting EID from a lab-based diagnostic service to an integrated clinic-based chronic care model. This model was piloted at 21 facilities. An evaluation is needed.

Keywords: Uganda, HIV, HIV-exposed infant, Early infant diagnosis, Pediatric HIV, Prevention of mother-to-child transmission, Testing, Retention, Linkage to care

\footnotetext{
* Correspondence: vijay.narayan.5@gmail.com

${ }^{2}$ Clinton Health Access Initiative, Kampala, Uganda

Full list of author information is available at the end of the article
}

(c) The Author(s). 2018 Open Access This article is distributed under the terms of the Creative Commons Attribution 4.0 International License (http://creativecommons.org/licenses/by/4.0/), which permits unrestricted use, distribution, and reproduction in any medium, provided you give appropriate credit to the original author(s) and the source, provide a link to the Creative Commons license, and indicate if changes were made. The Creative Commons Public Domain Dedication waiver (http://creativecommons.org/publicdomain/zero/1.0/) applies to the data made available in this article, unless otherwise stated. 


\section{Background}

Globally the number of infants newly infected with HIV has declined in recent years, from 370,000 in 2009 to 150,000 in 2015 [1, 2]. However, despite increasing access to anti-retroviral therapy (ART) in sub-Saharan Africa, diagnosis and treatment of HIV-positive (HIV+) infants has lagged behind [3, 4]. In Uganda, an estimated 91,000 HIV-exposed infants (HEI) - infants aged 018 months born to HIV+ mothers- were born in 2010, yet only $41,340(46 \%)$ were tested $[5,6]$. Out of 78,000 total HIV+ children (aged 0-14 years) who were eligible for ART in Uganda in 2010, only 24,031 (31\%) were diagnosed and started on ART [6].

$\mathrm{HIV}+$ infants must be diagnosed and treated early to survive. Unlike in adults, disease progression is rapid in $\mathrm{HIV}+$ infants [7]. For HIV+ infants not initiated on ART, $35 \%$ are likely to die within the first year of life and 52\% by 2 years of age [8]. Early initiation of HIV+ infants on ART slows disease progression, suppresses viral load, and dramatically reduces mortality rates [9-11]. Diagnosis of HEI is conducted in resource-limited settings by DNA Polymerase Chain Reaction (PCR) testing of Dried Blood Spot (DBS) samples [12, 13]. In Uganda, DBS samples are drawn from HEI at health facilities and referred to a specialized reference laboratory for DNA PCR testing.

As shown in Fig. 1, Early Infant Diagnosis (EID) is a complex process that has many points where HEI can be lost [14]. Since the diagnostic process for HEI can take several months, it is vital that all HEI receive regular clinical care (growth and developmental monitoring, clinical assessment) and Cotrimoxazole prophylaxis until their HIV status is confirmed. An HIV+ infant may become symptomatic from an infection before PCR results return. The onset of HIV clinical symptoms suggests advanced disease progression, and delaying treatment of symptomatic HIV+ infants increases risk of death $[15,16]$. Therefore all HEI should be visiting the clinic regularly so that any opportunistic and other HIV-associated infections can be immediately recognized and treated; the infant can then be immediately diagnosed based on clinical status and initiated on ART. Cotrimoxazole prophylaxis is critical; it has been shown to reduce mortality by $43 \%$ for HIV+ infants who have not yet started ART [17]. Caregivers of HEI require intensive and continuous counseling about the testing process and proper feeding practices [18].

In Uganda, after successfully getting EID systems up and running, the Ministry of Health $(\mathrm{MOH})$ initially focused on increasing the number of HEI tested and decentralizing EID services to a greater number of health facilities. PCR tests increased from 6437 in 2007 to over 37,000 in 2009 [5, 6]. However, little attention was paid to HEI outcomes after testing. No data was available at national level on whether HEI received test results and completed the testing algorithm, and whether HIV+ infants were enrolled at an HIV/ART Clinic, initiated on ART and active in care.

To improve retention of tested HEI, Uganda's $\mathrm{MOH}$ and the Clinton Health Access Initiative (CHAI) piloted a transport refund program at three regional referral hospitals in mid-2008. Caregivers of HEI were provided

\section{EID Continuum of Care in Uganda}

Health Facility

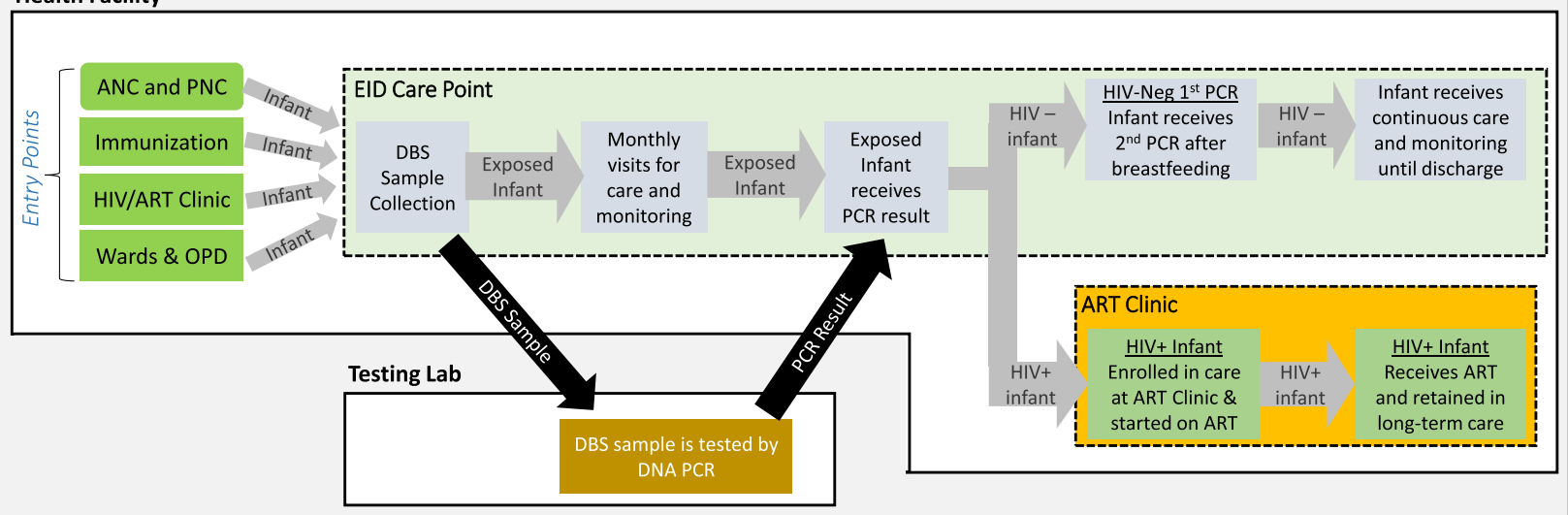

Fig. 1 EID Continuum of Care in Uganda. Diagram showing steps in the HIV infant diagnosis continuum: HEl encounter the health facility at many 'entry point' clinics. Health workers (HWs) at entry points must proactively screen for HIV exposure and link the identified HEl to the facility's testing point for DBS sample collection. The DBS sample must be dispatched to the reference lab, tested by DNA PCR, and result returned to the health facility by the time the HEl caregiver returns for their next appointment. If the PCR result is negative, the HEl must receive a 2nd confirmatory PCR test 6 weeks after cessation of breastfeeding. If the infant tests $\mathrm{HIV}+$, the infant must be immediately enrolled at the HIV/ART Clinic and initiated on ART. ANC = Antenatal Clinic; PNC=Postnatal Clinic; ART = Antiretroviral Therapy; OPD = Outpatient Department; DBS = Dried Blood Spot; PCR = Polymerase Chain Reaction 
transport reimbursement at each visit to encourage and enable them to return to the health facility to receive PCR test results and follow-up care. The cash amounts varied depending on how far the mother traveled.

The $\mathrm{MOH}$ and $\mathrm{CHAI}$ conducted this study at 7 health facilities to achieve three objectives: quantify the retention outcomes of tested HEI at 3 hospitals, identify the factors driving HEI loss to follow-up at each point in the EID continuum of care, and assess the effect of the transport refund pilot on HEI retention.

\section{Methods}

This mixed-methods study was conducted at 7 government health facilities in Uganda- retrospective cohort review of tested HEI at 3 regional referral hospitals, and qualitative assessment at all 7 health facilities. The selected facilities cut across different health facility levels and geographic regions (Table 1).

The retrospective patient cohort review tracked retention outcomes of all HEI aged 6 weeks to 18 months tested by DNA PCR between September 2007 and February 2009 ( $n=1268 \mathrm{HEI})$. To identify the facility-level factors driving loss-to-follow-up of HEI, qualitative assessment of EID program implementation was done using 2 methods: semi-structured interviews with HWs and structured observation of health facility systems. Ethical approval for this study was obtained from the Mildmay Uganda Research and Ethics Committee.

\section{Data collection and analysis for cohort review}

All DNA PCR tests of HEI in Uganda were captured in a national electronic database housed at the $\mathrm{MOH}$ EID office. After a sample was tested by DNA PCR at the regional reference lab, a copy of the result was sent to the MOH EID office and captured in a Microsoft Excel database. At the outset of this study, a list of HEI who received a DBS test at the 3 hospitals during the review period was extracted into a separate Excel database for this study. The extract from the national EID database provided the date of DBS test, age at DBS test, and HIV test result for each HEI in the cohort.
The retention data for each HEI was then manually collected at each hospital from $10+$ data sources spread across several different clinics. Data was collected on every follow-up visit made by each HEI in the cohort and all other variables of interest (Table 2) and inputted into the study database. In addition to the HEI cohort review, we collected data at 1 hospital on the number of HIV+ pregnant women whose infants were tested after birth, which provided a measure of HEI testing coverage.

The data was analyzed using Excel and SPSS software. 95\% confidence intervals were calculated for mean values of age at testing, enrollment into care, and ART initiation. Statistical significance of retention changes before and after the transport refund intervention was assessed using a z-test for comparison of proportions in two independent samples $(\alpha=.05)$.

\section{Qualitative assessment: Data collection, analysis, and triangulation}

The qualitative assessment of EID implementation was designed to identify the factors contributing to HEI loss-to-follow-up. Study investigators conducted semistructured interviews with $37 \mathrm{HWs}$ across the 7 facilities to elicit information on practices and systems in EID program implementation, as well as capture their experiences and perspectives as direct implementers. Investigators conducted structured non-participant observation of EID implementation systems at the different clinics involved in EID within each facility (e.g. entry point clinics, lab, HIV clinic). Whereas the observations provided direct examination of EID practices and systems (e.g. flow mapping), the interviews provided information and context through the lens of the HW.

The interviews and observations captured how, when, where and by whom EID services were provided at the health facility; the flow of caregivers, samples, test results, and data; the data management and referral tools in use; and the package of care and counseling services provided to HEI. Topical guides were used for the interviews and observations, and the data was recorded in field note format. For each method, the raw qualitative data was typed into

Table 1 Health facilities in this study

\begin{tabular}{|c|c|c|c|c|}
\hline Facility Name & Facility Level & Region & Cohort Review? & Qualitative Assessment? \\
\hline Masaka Hospital & Regional Referral Hospital & Southwest & Yes & Yes \\
\hline Jinja Hospital & Regional Referral Hospital & East & Yes & Yes \\
\hline Lira Hospital & Regional Referral Hospital & North & Yes & Yes \\
\hline Kayunga Hospital & District Hospital & East-Central & No & Yes \\
\hline Wakiso H/C IV & Health Center IV & Central & No & Yes \\
\hline Mukono H/C IV & Health Center IV & East-Central & No & Yes \\
\hline Namayumba H/C IV & Health Center IV & Central & No & Yes \\
\hline
\end{tabular}

The five levels of the health system are 1) Regional Referral Hospital, 2) District Hospital, 3) Health Center IV, 4) Health Center III, and 5) Health Center II. During the review period, EID and pediatric ART services were mostly provided at the level of 'Health Center IV' and above 
Table 2 Key variables of interest and data sources at the hospital

\begin{tabular}{|c|c|c|}
\hline Variables & Data Sources & Clinics within Health Facility \\
\hline - Dates of each HEl follow-up visit & - DBS Dispatch Register & - Lab \\
\hline - Purpose of visits & - EID Follow-Up Cards & - ART Clinic \\
\hline - Whether test results received & - EID Data Register & - Antenatal Clinic \\
\hline -When test results received & - Transport Refund Register & - Postnatal Clinic \\
\hline - Breastfeeding status & - Test result slips for HEl & - Immunization Clinic \\
\hline - Provision of Cotrimoxazole prophylaxis & - Homemade clinic attendance registers & - Outpatient Department \\
\hline - Clinical care indicators & - Homemade HEI clinic attendance registers & - Feeding Units \\
\hline - When due for 2nd PCR & - Homemade follow-up register for HIV-exposed infants & - Pediatric Ward \\
\hline - Whether transport refund given & - Antenatal, Postnatal \& Immunization Registers & - Maternity Ward \\
\hline - Dates of sample dispatch and result return to facility & - Pediatric Ward Register & - Adult Wards \\
\hline - Referring clinic (entry point for HEl) & - Outpatient Register & \\
\hline - HIV+: If/when enrolled at ART clinic & - Pre-ART \& ART Registers & \\
\hline - HIV+: Dates of each visit at ART clinic & - Patient files for HIV+ patients & \\
\hline \multicolumn{3}{|l|}{ - HIV+: If initiated on ART and when } \\
\hline - HIV+: CD4 tests and clinical stage & & \\
\hline
\end{tabular}

HEI HIV-exposed infant, PCR Polymerase Chain Reaction, ART Antiretroviral Therapy, DBS Dried Blood Spot, EID Early Infant Diagnosis

Microsoft Word, collated by topic and question, indexed, and analyzed to identify common themes. The analyses from direct observation data and interview data were triangulated [19]; we first generated broader categories of factors affecting testing and retention of HEI, then identified specific factors within each category, and finally broke down the factors by which part(s) of the EID continuum they affected.

The interviews also assessed HW knowledge and awareness of EID and pediatric ART (how to screen for HIV exposure, signs of pediatric HIV infection, testing and feeding algorithms, infant ART initiation criteria), as well as the counseling messages conveyed to caregivers. To obtain a basic measure of $\mathrm{HW}$ knowledge, we calculated how many of the interviewed HWs provided accurate information about each topical area.

We used both interviews and observations to assess whether and how consistently clinical care and counseling were provided to HEI. Given the lack of clinical care and counseling data for individual HEI visits, our assessment focused more broadly on whether each key component of HEI clinical care and counseling package was provided as 1) routine practice at every visit, with few exceptions, 2) provided occasionally or only when triggered for a specific reason, or 3) not provided at all, except in rare situations. To assess the messaging provided by HWs to caregivers during counseling (as a proxy measure for counseling 'quality'), we compared the information conveyed by HWs with the counseling messages prescribed in the national EID training manual [20].

\section{Results}

Outcomes for HIV-exposed and HIV-positive infants

This study tracked retention outcomes of 1268 HEI who received a DNA PCR test at 3 regional referral hospitals in Uganda between September 2007 and February 2009. 244 out of 1268 HIV-exposed infants tested HIV-positive (19.2\%).

\section{Identification and testing of HEI}

The number of HEI in the catchment area of the three hospitals was unknown. However, the number of HIV+ pregnant women receiving Prevention of Mother-to-Child Transmission (PMTCT) services at the hospitals served as a reasonable proxy for the minimum number of HEI needing to be tested. At one hospital, only 18\% (67/367) of HIV+ pregnant women brought their infants back for DBS testing after birth. In addition, analysis of qualitative observation and interview data revealed that HWs at many other entry points (e.g. outpatient department, immunization, feeding units, wards) were not proactively screening infants for possible exposure to HIV, nor taking active steps to ensure that identified HEI successfully reached the EID testing point.

\section{Age at testing for HEI}

The mean age of first DBS test was 7.0 months of age among the 1268 HEI who were tested at the 3 hospitals $(\mathrm{SD}=5.9)$. (Table 3) Only $24.1 \%$ were tested at $\leq 2$ months of age. Among infants testing $\mathrm{HIV}+$, the mean age of first DBS test was 9.6 months $(n=244$, SD $=7.3$ ), mean age of enrolment into care was 13.9 months 
Table 3 Age at testing, enrollment and ART initiation for HIVexposed and infected infants

\begin{tabular}{lll}
\hline All HIV-Exposed Infants & $\begin{array}{l}\text { Mean Age in Months } \\
(95 \% \mathrm{Cl}), \mathrm{SD}\end{array}$ & $\mathrm{N}$ \\
Age at 1st DBS Test & $7.0(6.7-7.3), \mathrm{SD}=5.9$ & 1268 \\
HIV+ Infants Only & & \\
Age at 1st DBS Test & $9.6(8.7-10.5), \mathrm{SD}=7.3$ & 244 \\
Age at enrollment in & $13.9(11.7-16.0), \mathrm{SD}=10.4$ & 86 \\
ART Clinic & $16.1(11.6-20.6), \mathrm{SD}=11.9$ & 27 \\
Age at ART initiation &
\end{tabular}

DBS Dried Blood Spot, ART Antiretroviral Therapy, Cl Confidence Interval, SD Standard Deviation

$(n=86, \mathrm{SD}=10.4)$ and mean age of ART initiation was 16.1 months $(n=27, \mathrm{SD}=11.9)$.

\section{$H E I$ receiving test results and completing testing algorithm} Only $57 \%$ of the 1268 HEI received their PCR test results. Out of the 261 HEI who had a negative 1st PCR at less than 6 months of age and received their results, only $18.4 \%$ had a confirmatory 2nd PCR test. Breastfeeding status of HEI was not documented making it difficult to know the exact number requiring a confirmatory PCR test, but according to national estimates, greater than $90 \%$ of HIV+ mothers in Uganda breastfed during the review period $[6,21]$.

\section{Effect of transport refund pilot on HEI receiving test results} Figure 2 shows the percent of caregivers receiving PCR results among those receiving at least one transport refund (post-intervention period of May 2008 - February 2009) and those never receiving a refund (pre-intervention period of September 2007-April 2008). For the three hospitals in aggregate, retention increased from $54 \%(n=763)$ to $58 \%$ $(n=505)(p=.08)$. The individual hospitals had increases of $13 \%(p=.01), 0 \%(p=.50)$ and $13 \%(p=.02)$. Even when considering only the 2 hospitals with a statistically significant gain in retention, the percent receiving results was still under $70 \%$ for both. The average cost of the program per HEI was 21 USD (average of 7 USD per visit, and 3 visits per HEI).

\section{Outcomes for HIV+ infants}

Figure 3 shows the outcomes for the 244 infants testing $\mathrm{HIV}+$ at the 3 hospitals. Out of 244 infants testing HIV + , only 150 returned to the health facility and received results (39\% loss). Out of the $150 \mathrm{HIV}+$ infants receiving results, 98 enrolled into care at the HIV/ART Clinic (35\% loss). Finally, 57 of the 98 who enrolled at the HIV/ART Clinic were still alive and active in care as of January 2010 (42\% loss). Overall, only $23.4 \%$ of HIV+ infants diagnosed through the EID program were still active in care. 75 of the $98 \mathrm{HIV}+$ infants enrolled in care at the HIV/ART Clinic were eligible for ART under national guidelines during the review period, but only $36 \%(27 / 75)$ were initiated on ART.

Drivers of HEI loss throughout the EID continuum of care Synthesis, analysis and triangulation of qualitative data from the HW interviews and direct clinic observations yielded 6 categories of factors contributing to HEI loss, as well as the specific factors within each category and which part(s) of the EID continuum they affected (Table 4). The 6 categories included EID clinic systems

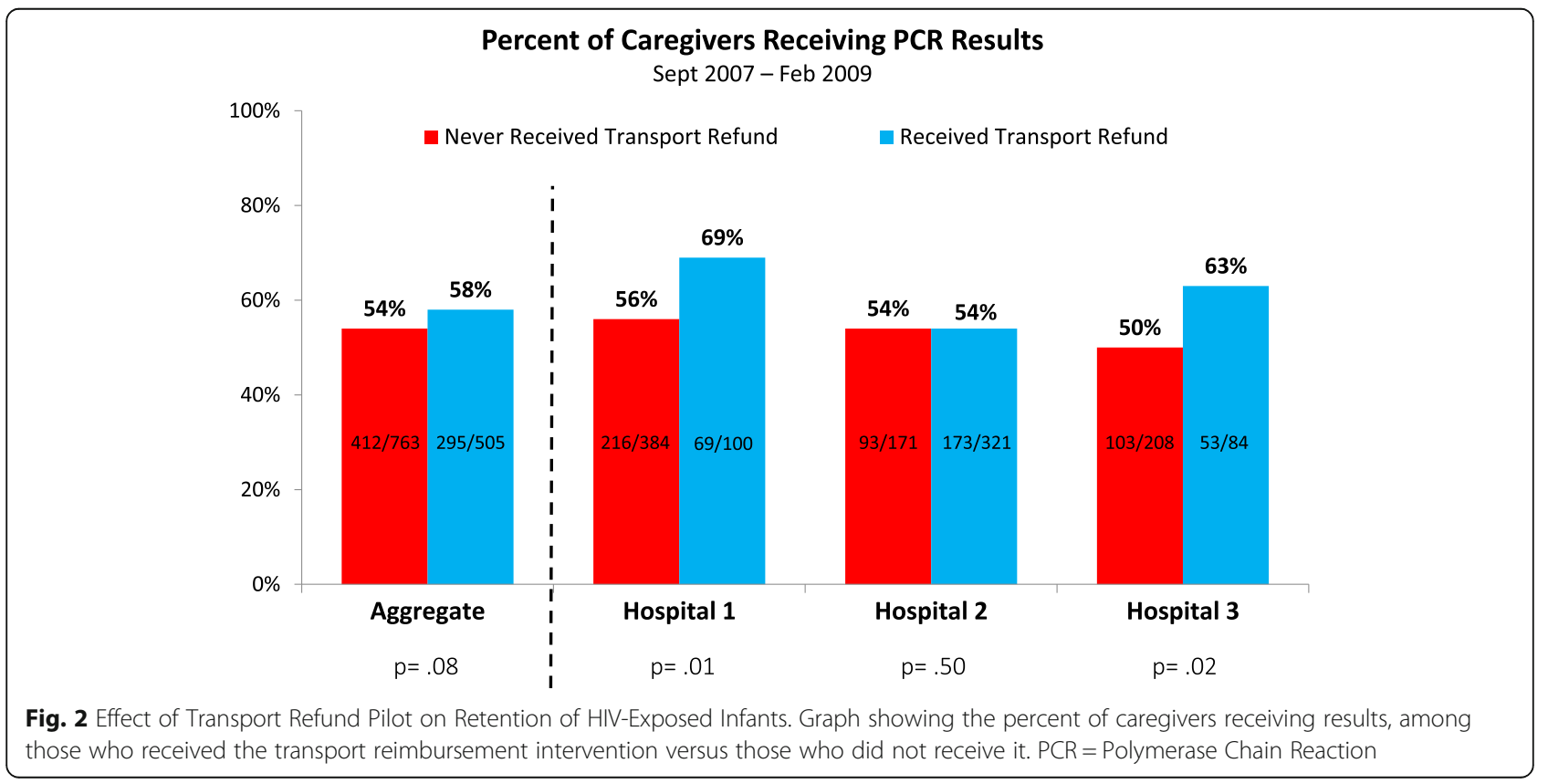




\section{Retention Outcomes for Infants Testing HIV-Positive \\ 3 Regional Referral Hospitals \\ Sept 2007 - Feb 2009}

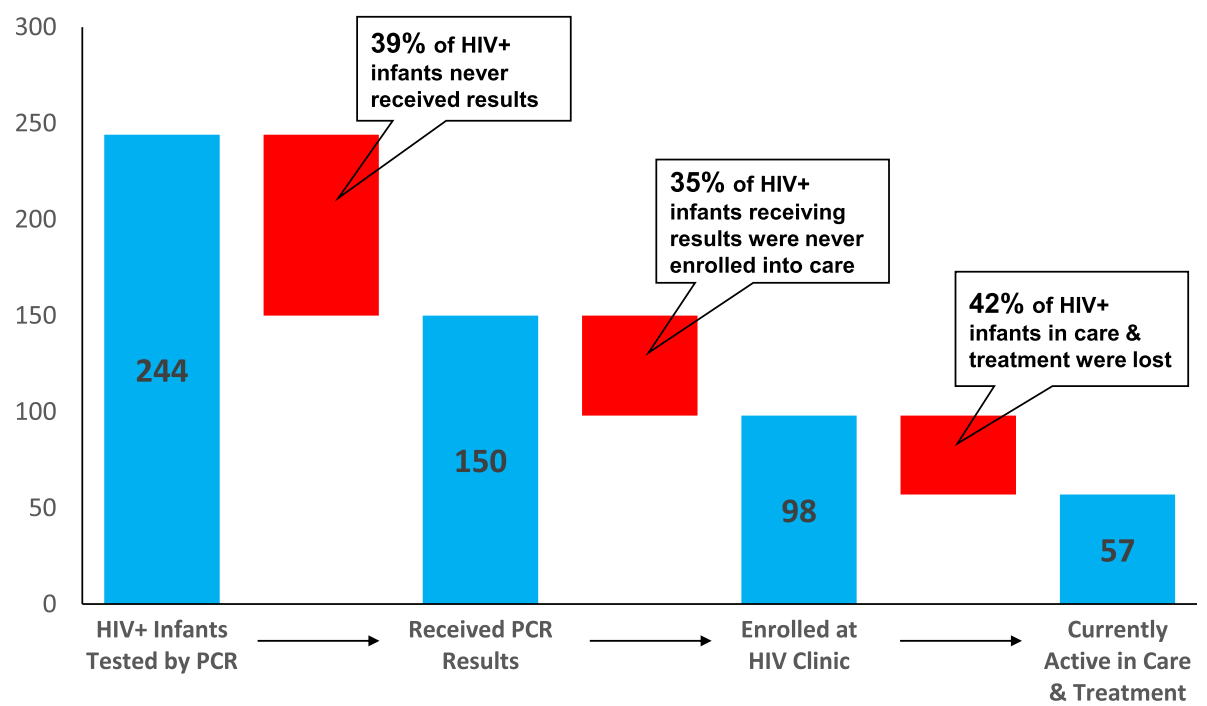

Fig. 3 Retention Outcomes for Infants Testing HIV-Positive. Graph showing the outcomes for HIV-exposed infants testing HIV-positive by DNA PCR. The red bars represent the number of infants lost-to-follow up at each step in the EID process. PCR = Polymerase Chain Reaction

and patient flow; referral and data management systems; clinical care and visit schedule; counseling materials, messages and techniques; knowledge and awareness of health workers, and DBS sample-result turnaround time.

\section{Drivers of loss: EID clinic systems}

An example of the clinic system from a reviewed facility is shown in Fig. 4. Most facilities had an ad hoc and fragmented EID clinic system - particularly the flow of caregivers, samples, and test results - that contributed to HEI getting lost throughout the EID continuum. Health facilities did not have one, clear, well-equipped place where all HEI caregivers would go for registration and post-test counseling on the day of the DBS test, and then return to receive results and follow-up care. Instead, at some facilities DBS testing was decentralized to entry point clinics, but all results were given from the main lab. At other facilities, all HEI were referred to the main lab for the DBS test, but result distribution was decentralized to the entry point where the HEI was identified. Most often, however, it was a combination of the two systems, with HEI receiving a DBS test and results at different places within the same facility depending on the entry point clinic where they were identified; consequently, HEI caregivers and HWs often didn't know where PCR results were given. Data management, follow-up, care provision and counseling of HEI were fragmented across different clinics.
At one hospital, test results were returned to caregivers at three different places depending on where HEI initially received their DBS test (Fig. 5): 71\% of the HEI tested and followed up at the ART Clinic received results $(n=241)$, compared to $49 \%$ of HEI tested and followed up at the Maternal-Child Health Clinic $(n=151)$ and $41 \%$ of HEI tested and followed up at the pediatric ward $(n=24)(p$ $<.0001$ for comparison of ART Clinic versus other points). Interviewed HWs at all three units of the hospital expressed that the ART clinic was the best location for providing PCR results and all follow-up care; they noted that because $\mathrm{HIV}+$ mothers were already attending the ART Clinic monthly for their own chronic care, having HEI follow-up visits there allowed both mother and baby to receive care simultaneously, making it more likely that the infant would receive regular care and PCR results.

At several reviewed facilities, HEI caregivers received results at the facility's laboratory. Several HWs noted that the lab was a centralized place for giving results and often most convenient for the facility, but that it was a poor location for result delivery because laboratory technicians in Uganda are not professionally qualified to provide sensitive post-test counseling to HEI caregivers, and also because the lab cannot not provide care or follow-up of HEI.

\section{Drivers of loss: Referral systems and data management tools}

Lack of mechanisms for referral within the health facility contributed to HEI loss at multiple points in the EID 


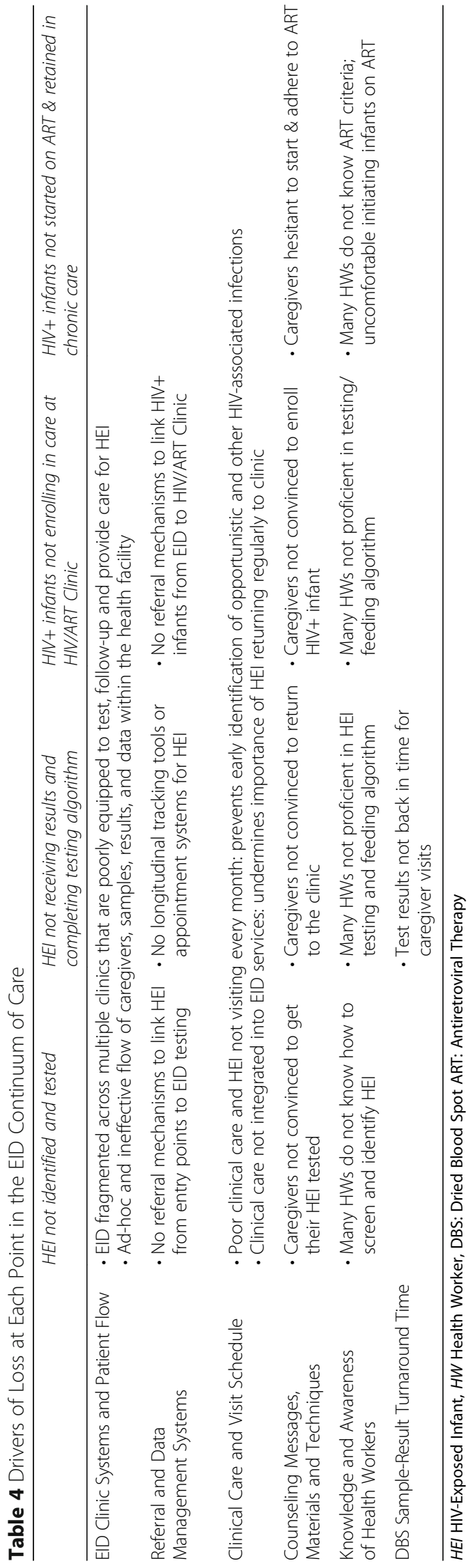




\section{Fragmented EID Clinic System at One Health Facility}
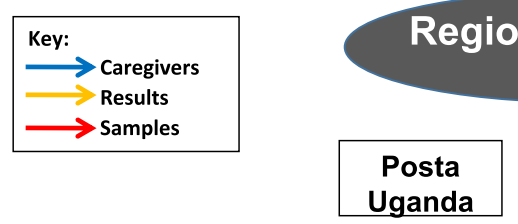

Region

\section{Lab}

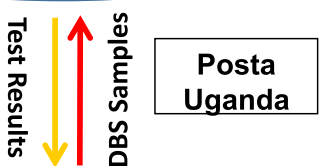

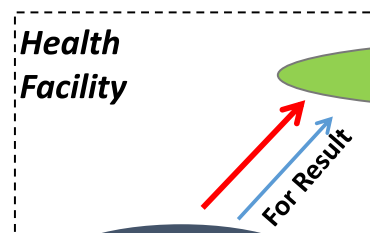

Antenatal Clinic (ANC)

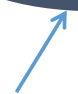

\section{Health Facility Lab}

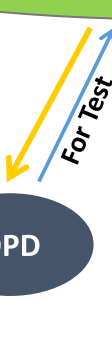

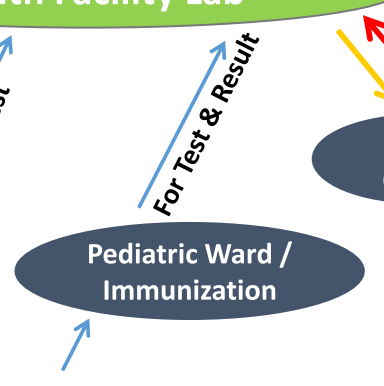

ART

Clinic

\section{EID Clinic System}

- HEl identified at ANC: receive DBS test at ANC, but receive results at Lab

- HEl identified at OPD: receive DBS test at Lab, but receive results at OPD

- $\underline{\text { HEl identified at Pediatric Ward or }}$ Immunization Clinic: receive DBS test and results at Lab

- HEl identified at ART clinic: receive both DBS test and results at ART clinic

- Only the ART Clinic tracks \& follows up HEI who don't receive results

- Each clinic provides different levels of care and counseling to $\mathrm{HEI}$

Fig. 4 Fragmented EID Clinic System at One Health Facility. Diagram depicting the flow of caregivers, samples, and results at one of the reviewed health facilities. ANC = Antenatal Clinic, DBS = Dried Blood Spot, OPD = Outpatient Department, HEI=HIV-Exposed Infant

continuum of care: linkage of HEI from entry points to testing point, and linkage of HIV+ infants from EID services to the HIV/ART Clinic. The earliest point of HEI identification is when the HIV+ mother is still pregnant and receiving PMTCT services in the facility's antenatal clinic. However, there was no system in place for the antenatal clinic to provide documentation to the EID testing point, preventing the EID testing point from knowing that there would be an HEI needing a DBS test after delivery and in what month, and following up in the community if the HEI didn't come. Similarly, when HEI were identified at entry points such as the outpatient department and immunization clinic, caregivers were told verbally to attend the EID testing point but no information was given to the EID testing point. Likewise, after HIV+ infants received their PCR results, the ART

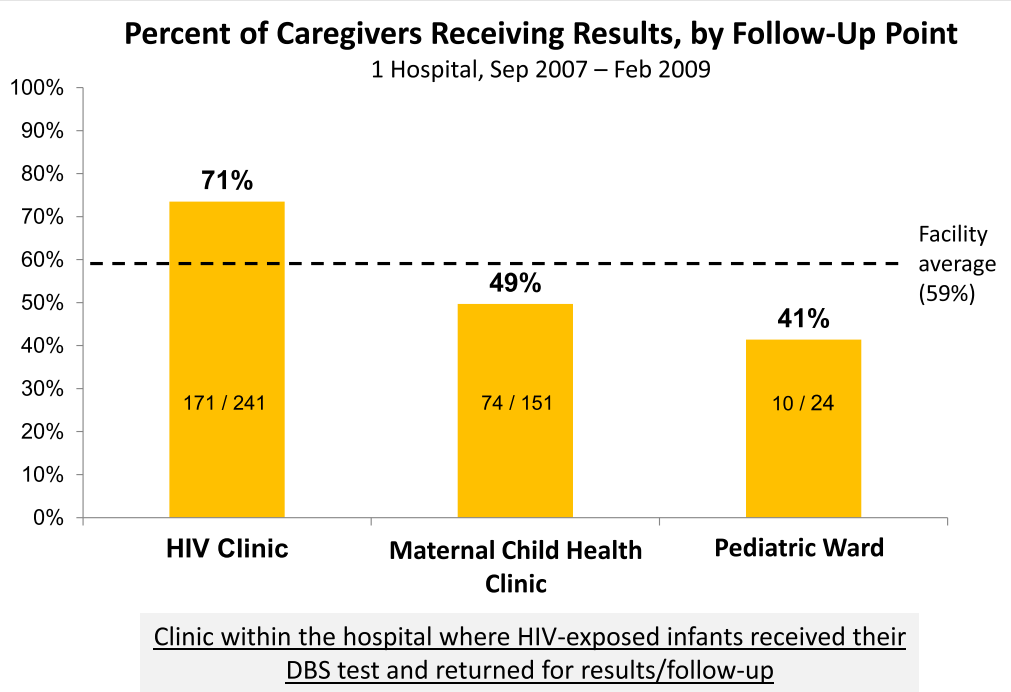

Fig. 5 Relationship between "location of HIV-exposed infant follow-up" and "percent of caregivers receiving PCR results". Graph showing the percent of caregivers receiving results at 1 hospital, by testing/follow-up point 
Clinic was not informed that an HIV+ infant needed to be enrolled and did not have any contact information.

Poor data management systems and tracking tools contributed to HEI not receiving results and failing to complete the testing algorithm. There was no system to document and manage appointments for clinic visits, which prevented HWs from identifying the HEI who did not return to the health facility and following them up by phone or home visit. The data register for HEI captured only the first visit when the DBS test was done. Because the register did not capture any follow-up visits, HWs didn't know where HEI were in the testing algorithm - e.g. whether they had received 1st PCR results, whether they were still breastfeeding, and when they were due for a 2 nd PCR test.

\section{Drivers of loss: Clinical care, prophylaxis, and counseling}

Among the 7 reviewed facilities, clinical care wasn't provided to HEI as routine practice, and barely at all in many cases (Table 5). Only 3/7 facilities provided Cotrimoxazole as routine practice at every HEI visit, only $3 / 7$ measured weight regularly, none measured height or monitored developmental milestones. 5/7 facilities regularly conducted a clinical assessment for HIV signs and symptoms. Clinical care was not integrated into EID services and there was no standard HEI visit schedulecaregivers visited the clinic only for the purpose of receiving DBS tests or results. EID wasn't viewed by HWs and caregivers alike as a chronic care service in which routine visits were essential for HEI survival.

Also shown in Table 5, counseling was inconsistently provided to HEI caregivers on the testing process, feeding, and nutrition- during pregnancy, after birth at the identification/referral point, and at the EID testing point. In addition to inconsistency of counseling provision, quality was an issue. Analysis of data from observation of counseling sessions and HW interviews revealed that HWs were not conveying many of the key messages needed to adequately educate mothers about why they needed to get their babies tested, the diagnostic process, and steps to keep their baby alive and healthy.

HWs reported that they had not been adequately trained on techniques to effectively convey the key information, noting the particular challenges of counseling in Uganda's HIV context. These gaps were compounded by the absence of counseling reference materials for HWs and informational materials for HEI caregivers; some HWs pointed out that similar materials were effective in other non-HIV programs.

\section{Drivers of loss: Knowledge and awareness of health workers}

Many HWs lacked sufficient knowledge and awareness about EID and pediatric HIV. We found that HWs weren't proactively checking exposure status and actively identifying HEI at many entry points, stemming from insufficient knowledge (27/37 weren't proficient with the process for screening infants of HIV + mothers for HIV exposure) and poor participation (interviewed HWs did not always perceive proactive screening as one of their core job functions). 30/37 HWs could not list more than two common clinical signs of pediatric HIV infection, and HWs at entry points acknowledged that they may have failed to recognize clinical presentations as opportunistic or other HIV-associated infections. 29/37 interviewed HWs didn't know the full infant HIV testing

Table 5 Care and Counseling Provided to HIV-Exposed Infants at 7 Health Facilities

\begin{tabular}{|c|c|c|c|c|c|c|c|}
\hline & $\begin{array}{l}\text { Regional Referral } \\
\text { Hospital }\end{array}$ & $\begin{array}{l}\text { Regional Referral } \\
\text { Hospital }\end{array}$ & $\begin{array}{l}\text { Regional Referral } \\
\text { Hospital }\end{array}$ & $\begin{array}{l}\text { District } \\
\text { Hospital }\end{array}$ & $\begin{array}{l}\text { Health } \\
\text { Center IV }\end{array}$ & $\begin{array}{l}\text { Health } \\
\text { Center IV }\end{array}$ & $\begin{array}{l}\text { Health } \\
\text { Center IV }\end{array}$ \\
\hline Cotrimoxazole prophylaxis & $\sqrt{ } \bullet$ & $\checkmark \checkmark$ & $\checkmark \checkmark$ & $\checkmark \checkmark$ & - & - & $\checkmark \bullet$ \\
\hline Growth monitoring- weight & - & $\checkmark \checkmark$ & $\sqrt{\bullet}$ & $\checkmark \checkmark$ & - & - & - \\
\hline Growth monitoring- height & - & - & - & - & - & - & - \\
\hline $\begin{array}{l}\text { Immunization assessment } \\
\text { and referral }\end{array}$ & - & $\checkmark \bullet$ & - & $\checkmark \checkmark$ & - & - & - \\
\hline $\begin{array}{l}\text { Clinical assessment for } \\
\text { signs/symptoms of HIV }\end{array}$ & $\checkmark \bullet$ & $\sqrt{ } \bullet$ & $\checkmark \bullet$ & $\checkmark \bullet$ & $\checkmark \bullet$ & - & - \\
\hline Developmental assessment & - & - & - & - & - & - & - \\
\hline $\begin{array}{l}\text { Counseling on nutrition } \\
\text { and feeding practices }\end{array}$ & $\checkmark \bullet$ & $\checkmark \bullet$ & $\checkmark \bullet$ & $\checkmark \bullet$ & $\checkmark \bullet$ & - & - \\
\hline $\begin{array}{l}\text { Counseling on testing } \\
\text { algorithm and EID process }\end{array}$ & $\checkmark \bullet$ & $\checkmark \bullet$ & $\checkmark \bullet$ & $\checkmark \bullet$ & $\checkmark \bullet$ & - & - \\
\hline
\end{tabular}

KEY:

$\checkmark \checkmark$ Always provided: provided as routine practice at every clinic visit, with rare exceptions

$\checkmark$ Sometimes provided: provided occasionally, ad hoc, or only when triggered for specific reason(s)

- Rarely or never provided: not provided at all except in rare situations

Assessment of clinical care provided to HIV-exposed infants and counseling of their caregivers. Results were derived from analysis and triangulation of qualitative data from direct observation of HIV-exposed infant clinic visits and interviews with health workers 
algorithm and proper feeding guidelines, likely contributing to substandard counseling of caregivers.

We found that many interviewed HWs (29/37) didn't know the correct ART initiation criteria. Further, several clinical staff working in ART clinics reported that they didn't want to initiate children on ART because they weren't comfortable with the complicated treatment regimens for HIV+ children, noting that they were afraid to make fatal mistakes and needed more hands-on training to first build their confidence.

Most HWs reported not having been trained in EID recently or at all. Among those who were trained, several noted that the one-day EID trainings at the facility were not sufficient for them to retain the information and master the skills they were newly introduced to. HWs complained that there were no organized follow-up trainings and mentorships, and also noted the high rates of HW transfer and turnover as challenges. HWs also pointed out that the training did not include all of the topics discussed in our interviews, and some reported that they were never oriented on the most recently updated EID guidelines.

\section{Drivers of loss: Sample-result turnaround time}

Long sample-result turnaround time (TAT) also contributed to caregivers not receiving results- interviewed HWs expressed frustration that caregivers would come back 1 month after the DBS test only to find out that the result had not yet returned from the reference lab, and asserted that caregivers were disinclined to come back again because of this. Figure 6 shows TAT data from one of the hospitals. Test results took an average of 38 days to get back to the facility after the DBS sample was drawn ( $n$
$=222$ ), and an additional 31 days to be given to the caregiver (among the subset of caregivers who received results, $n=194$ ). Data from 2 of the hospitals shows that with larger sample-result TATs, fewer caregivers received results Fig. 7. However, even when PCR results came back within the desired range of $10-30$ days, less than $60 \%$ of caregivers ultimately received them $(n=155)$. Data from another hospital showed that among caregivers who didn't receive results, $73 \%$ did not make even a single follow-up visit $(n=201)$; TAT duration had no effect on this group of lost HEI.

\section{Discussion}

This study is the first-ever measure of longitudinal outcomes of HEI in Uganda. The findings are striking: only $40 \%$ of infants testing HIV+ ultimately enrolled at an ART clinic, and only $18 \%$ of all HIV+ infants were ultimately alive and retained in care. Only $57 \%$ of tested HEI received their 1st PCR test results. Among HEI with a negative 1st PCR, very few had a 2nd PCR, suggesting that transmission through breastfeeding was not being diagnosed. Mean age of testing was 7.0 months among all HEI, and 9.6 months among HIV+ infants, suggesting that many infected infants were captured only after they had already become sick. By such late ages, the disease may have progressed beyond the point of no return. Many HEI were not tested at all: only $18 \%$ of HIV+ pregnant women brought their HEI back for testing at 1 hospital, suggesting a major gap in linkage between PMTCT and EID services.

Lack of routine specialized care provision for HEI contributed to attrition of HIV+ infants throughout the EID continuum of care. While PCR results were pending, the

\section{Sample-Result Turnaround Time}

1 Hospital, Sep 2007 - Feb 2009

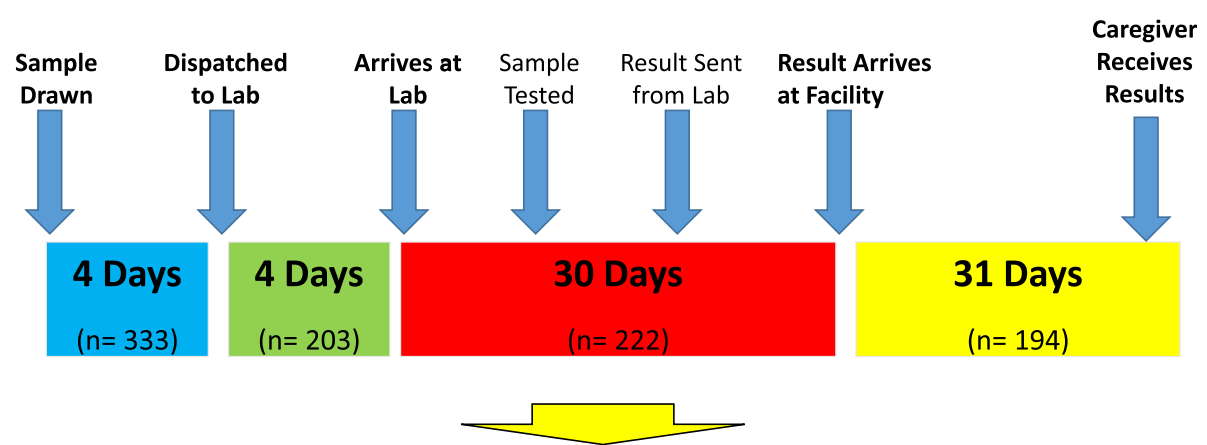

\section{On average, caregivers had to wait 69 days to receive DBS results}

Fig. 6 Sample-result turnaround time at one hospital. Diagram shows the average number of days it took for each step in the sample referral process. The $\mathrm{n}$ values differ for each point in the sample referral process because health workers inconsistently documented dates for 'sample draw', 'dispatched to lab' and 'arrives at lab' in facility and lab data registers. The $n$ values for 'result arrives at facility' and 'caregiver receives results' are different because not every tested infant received PCR results 


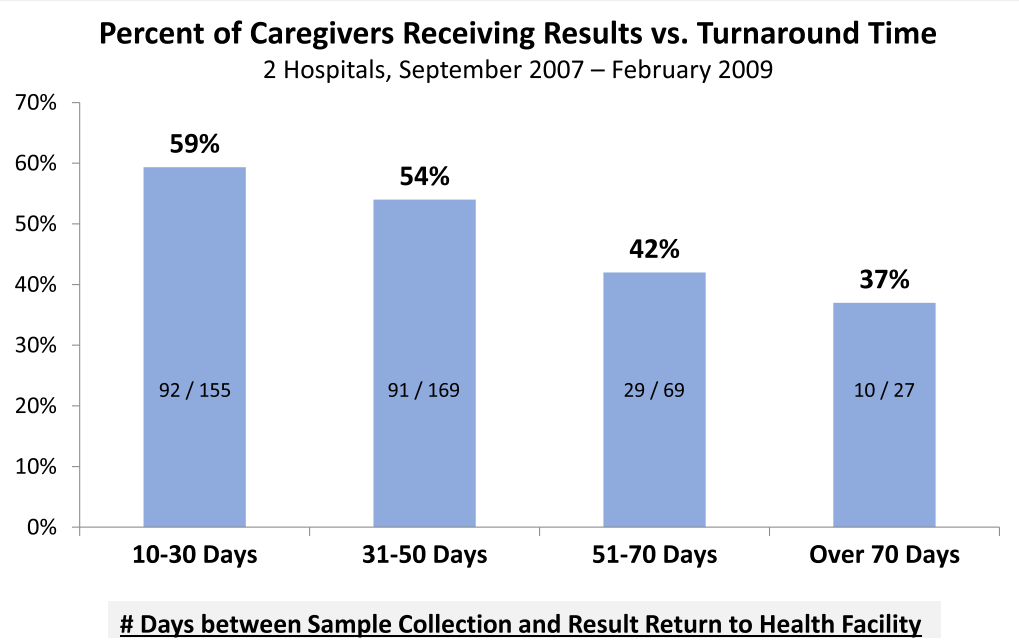

Fig. 7 Graph shows the percent of caregivers receiving PCR results as a function of sample-result turnaround time at 2 hospitals. The caregiver was due to return for test results 1 month after the DBS test, so the desired turnaround time for the EID program was in the range of '10-30 days'

infants who were HIV+ may have become symptomatic and required immediate ART. Since HEI were not coming to the clinic regularly for care check-ups and medication refills, clinical indicators of HIV infection may have been missed (e.g. weight loss, stunting); opportunistic or other HIV-associated infections may have gone untreated until it was too late. The absence of routine care undermined the importance of HEI returning to the facility regularly. If caregivers had been visiting the clinic monthly for care and counseling, it is likely they would have eventually received results at one of the visits and completed the testing algorithm, even when PCR results were delayed in getting back to the facility.

\section{Implications for Uganda's EID program and next steps}

Uganda's national program has succeeded in rapidly scaling up testing of HEI and has started to make inroads in

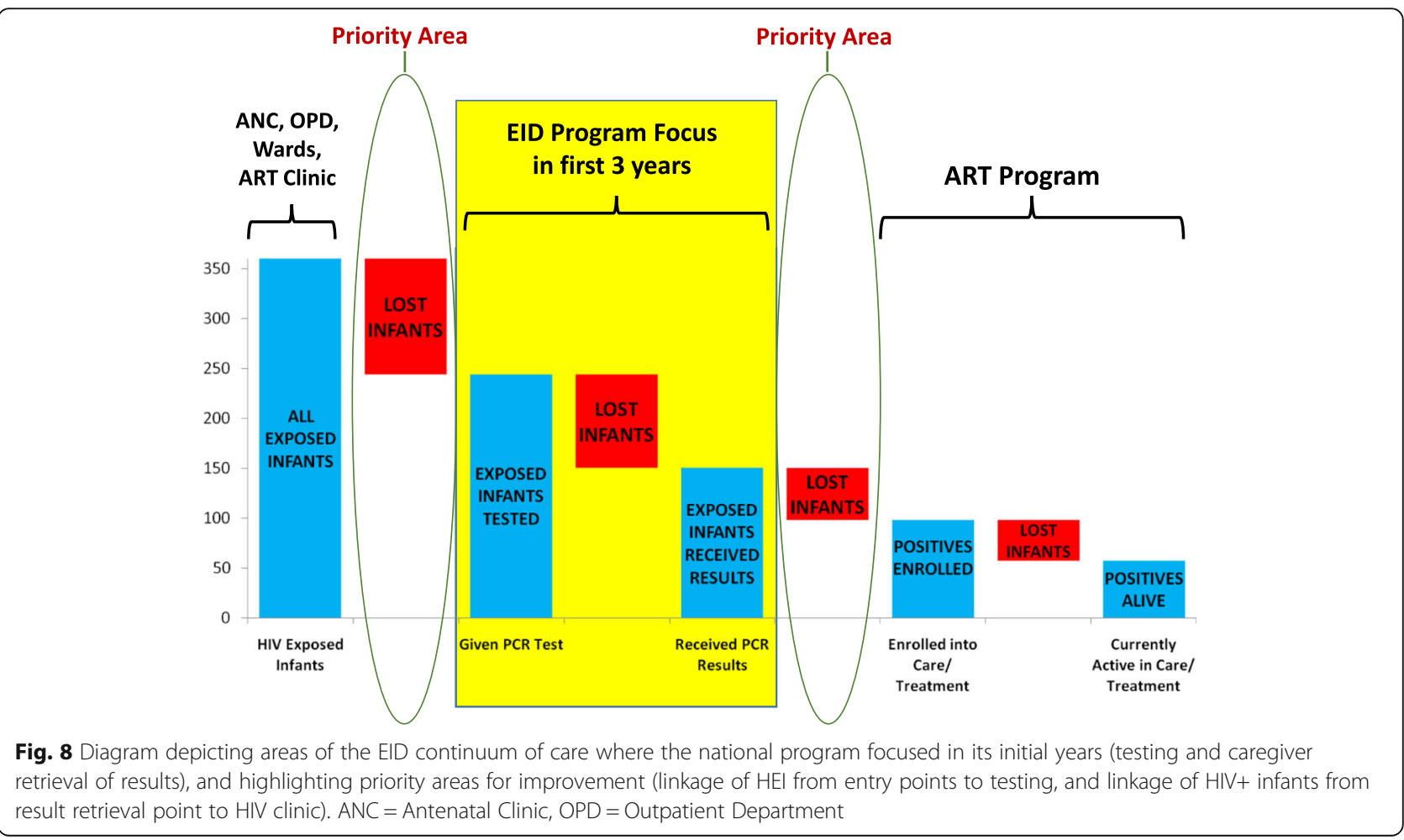




\section{New "EID Care Point" Model}

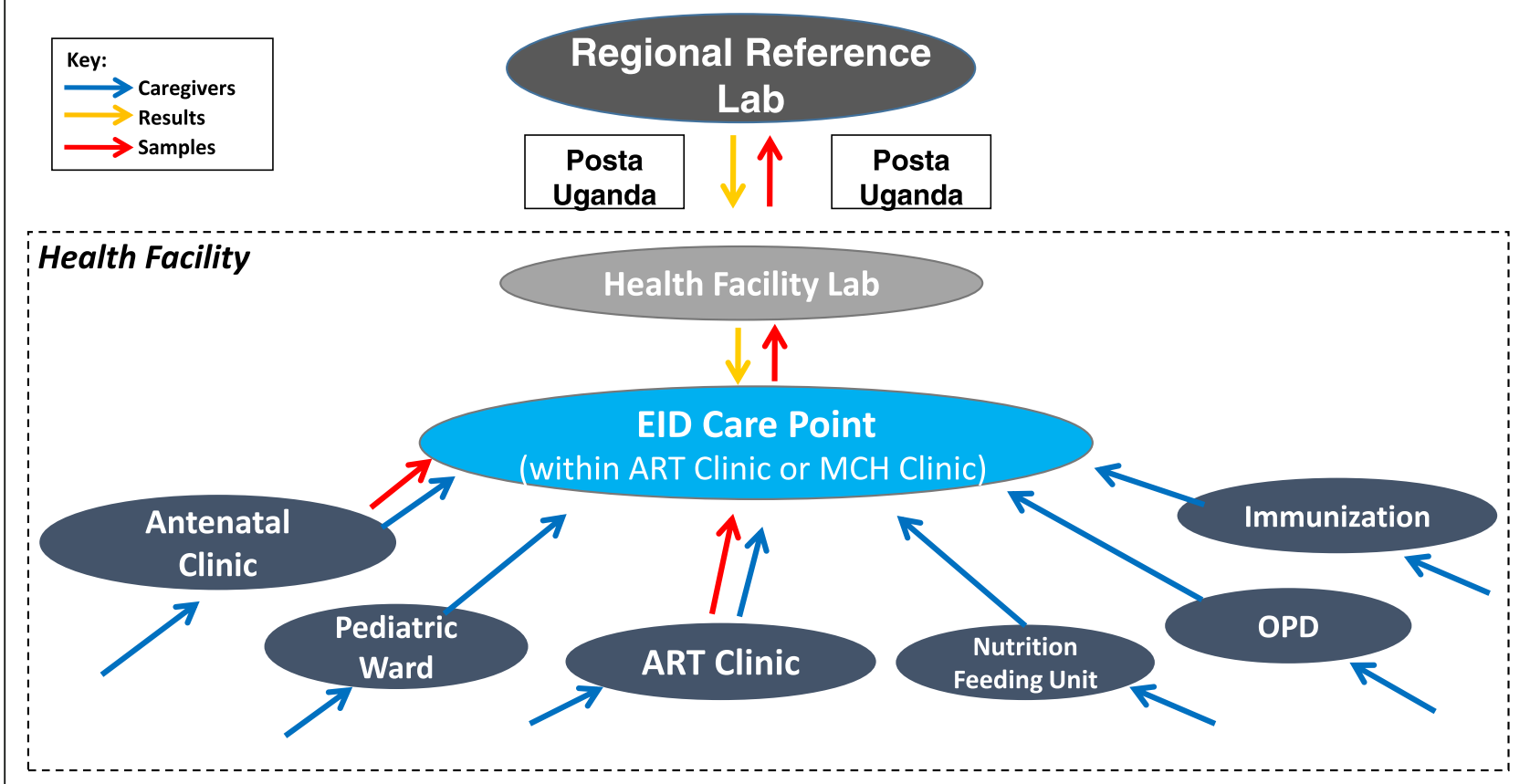

\section{DBS samples can be collected anywhere at health facility, but all HIV-exposed infants will visit the "EID Care Point" to receive PCR results, clinical care, counseling, and all follow-up services.}

Fig. 9 New "EID Care Point" Model. Diagram depicting the new proposed model for implementation of EID services at health facilities in Uganda. $\mathrm{DBS}=$ Dried Blood Spot, $\mathrm{PCR}=$ Polymerase Chain Reaction, EID = Early Infant Diagnosis

improving retention of HEI after testing. In addition to intensifying retention efforts, the program's focus should now expand to include linkage of HEI from entry points to DBS testing, and linkage of HIV+ infants to chronic care after receiving results Fig. 8. The study findings also underscore the need to improve EID's integration with PMTCT and pediatric ART services at national and district levels.

Findings from analysis of the transport refund pilot can help inform national program strategy. While more HEI caregivers received test results at 2 of the 3 hospitals among those who received the transport reimbursements, the overall improvement was modest and retention was still under $70 \%$ in the best case. This suggests that transport costs for caregivers, while one contributing factor, were not the only or biggest driver of HEI caregivers failing to receive results. Further, the high cost of implementing the program suggests challenges with sustainability and scalability of this type of intervention, even if the retention gains had been greater. Ultimately, addressing the underlying barriers to
HEI retention by improving health facility systems for how EID is implemented may provide a more feasible, cost-effective, and long-term solution.

Efforts to improve retention of tested HEI in Uganda have emphasized reduction of sample-result TAT. Indeed, the national EID program has undertaken several initiatives to reduce TAT, including creation of a new sample-result transport system and consolidation of the regional reference laboratories into a single, more efficient centralized national laboratory [22-24]. Our study found that sample-result TAT was too long and contributed to loss to follow-up, but even when TAT was less than 30 days, far too few caregivers received results $(<60 \%)$. Also, lower TAT wouldn't have a difference for the many lost HEI who never returned after the test. The key takeaway is that improving sample-result TAT needs to be one key component of a multi-faceted retention strategy, rather than the primary focus.

On a fundamental level, the EID program at facility-level needs to shift from a lab-based service to a 
clinic-based chronic care service. Based on this study's findings, we have developed a new model for EID implementation at health facilities, depicted in Fig. 9. In this model, HEI receive the full complement of EID servicesclinical care, counseling, registration and follow-up- at one centralized and well-equipped "EID care point" in the health facility. This "EID care point" sits within an existing clinic - ideally in the HIV/ART Clinic - and contains sufficient space, basic medical equipment, at least one dedicated clinical staff, data tools, and job aides to enable high-quality care, tracking, follow-up, and linkage of HEI. In contrast to the fragmented systems previously in place, all HEI are initially referred to the "EID care point" for registration and counseling, and return there for PCR results and all follow-up care visits. DBS tests can be done at the entry point clinics, "EID care point", or facility lab, but all patients, samples and results run through the "EID care point". The facility lab is a conduit for samples and results rather than the primary EID service delivery point. Each health facility adopts an EID clinic flow that works best for its context, but fulfills the minimum requirements of the "EID care point" model. The logic of the "care point" model lies in the premise that it is more effective to concentrate limited staffing and material resources for chronic care, counseling, tracking, and follow-up of HEI in one place, rather than scattering the focus and resources across many points in the facility.

To comprehensively address the gaps at each point in the EID continuum of care, the "EID care point" model is coupled with many other elements: instituting a routine monthly visit schedule for all HEI; establishing clear HEI clinical management protocols and providing clinical charts; setting up a triplicate referral system; providing medical equipment (e.g. height boards), longitudinal data registers, appointment books, counseling brochures and reference materials, job aides on HEI care, testing, and feeding, and financial support for follow-up of lost HEI. This set of initiatives is implemented through training and frequent on-site mentorship of health workers.

\section{Conclusions}

The study findings were disseminated within Uganda to relevant $\mathrm{MOH}$ departments, district health teams, health workers, NGO technical partners, donors, and international agencies (UN, WHO). Uganda's $\mathrm{MOH}$ and CHAI subsequently piloted the study recommendations (the "EID Systems Strengthening" program) at 21 health facilities, and the model was ultimately scaled up. An evaluation of the program model is critically needed to assess its impact and inform future strategies to improve HEI retention in Uganda.

\section{Abbreviations}

ART: Antiretroviral Therapy; CHAl: Clinton Health Access Initiative; DBS: Dried Blood Spot; DNA: Deoxyribonucleic Acid; EID: Early Infant Diagnosis; HEl: HIV-
Exposed Infant(s); HIV: Human Immunodeficiency Virus; HW: Health Worker; MOH: Ministry of Health; PCR: Polymerase Chain Reaction; PMTCT: Prevention of Mother-to-Child Transmission; TAT: Turnaround time

\section{Acknowledgements}

We would like to acknowledge several people who made this project possible. Sr. Lovis Kabagenyi (Jinja Hospital), Sr. Stella Apio (Lira Hospital), and Martha Ampumuza (Masaka Hospital) provided extensive support to the data collection team at each site. Deidre Thompson, Eleanor Joseph, and Evan Klaus of CHAI provided crucial technical support during the project. Rebecca Nakidde, the national EID administrator, helped administer the project. Dr. Zainab Akol (Program Manager of MOH AIDS Control Programme) and Dr. Godfrey Esiru (MOH PMTCT Program Manager) provided high-level government support for this study and its objectives.

\section{Funding}

This study was incorporated within the Clinton Health Access Initiative's technical support activities to the Ministry of Health AIDS Control Programme to improve national HIV Early Infant Diagnosis outcomes. The study was operational research and did not itself have specific designated funding.

\section{Availability of data and materials}

The data sets generated or analyzed during this study are available from the corresponding author on reasonable request.

\section{Authors' contributions}

CK oversaw the project. VN drafted the manuscript, designed the study protocol and tools, managed data collection, conducted data analysis, interpreted results \& formulated recommendations, developed dissemination presentations, and designed the resultant pilot program. IM \& CK developed the conceptual framework, designed study protocol, disseminated findings, and supported on pilot design. AK, JG, PE, LK, and MP assessed and interpreted the findings, and contributed to pilot development. All authors reviewed and approved the manuscript.

\section{Ethics approval and consent to participate}

This study was reviewed and approved by the Mildmay Uganda Research and Ethics Committee (MUREC). Confidentiality of patients in the retrospective cohort was ensured through use of serial identifier numbers in data capture tools. Informed written consent was obtained from all interviewed health workers.

\section{Consent for publication}

Not applicable.

\section{Competing interests}

The authors of this study declare that they have no competing interests.

\section{Publisher's Note}

Springer Nature remains neutral with regard to jurisdictional claims in published maps and institutional affiliations.

\section{Author details}

${ }^{1}$ Ministry of Health AIDS Control Programme, Kampala, Uganda. ${ }^{2}$ Clinton Health Access Initiative, Kampala, Uganda. ${ }^{3}$ Baylor College of Medicine, Kampala, Uganda.

Received: 20 November 2017 Accepted: 26 July 2018

Published online: 22 August 2018

\section{References}

1. UNAIDS. Global Report: UNAIDS Report on the Global AIDS Epidemic. 2010. Geneva, Switzerland. Accessed from http://www.unaids.org/globalreport/ documents/20101123_GlobalReport_full_en.pdf.

2. UNAIDS. Fact Sheet: Global HIV Statistics. 2017. Geneva, Switzerland. Accessed from http://www.unaids.org/sites/default/files/media_asset/ UNAIDS_FactSheet_en.pdf.

3. UNAIDS. 90-90-90 An Ambitious Treatment Target to Help End the AIDS Epidemic. 2014. Geneva, Switzerland. Accessed from http://www.unaids.org/ sites/default/files/media_asset/90-90-90_en_0.pdf.

4. Essajee S, Bhairavabhotla R, Penazzato M, et al. Scale-up of early infant HIV diagnosis and improving access to pediatric HIVcare in global plan 
countries: past and future perspectives. J Acquir Immune Defic Syndr. 2017; 75(suppl 1):S51-8.

5. UNAIDS, Uganda AIDS Commission. Uganda HIV Prevention Response and Modes of Transmission Analysis. 2009. Kampala, Uganda. Accessed from http:// web.worldbank.org/archive/website01390/WEB/IMAGES/UGANDAMO.PDF.

6. Government of Uganda. Uganda UNGASS Progress Report. March 2010; Kampala, Uganda. Accessed from http://data.unaids.org/pub/report/2010/ uganda_2010_country_progress_report_en.pdf.

7. Dunn D. Short-term risk of disease progression in HIV-1-infected children receiving no antiretroviral therapy or zidovudine monotherapy: a metaanalysis. Lancet. 2003;362(9396):1605-11.

8. Newell ML, Coovadia H, Cortina-Borja M, Rollins N, Gaillard P, Dabis F. Mortality of infected and uninfected infants born to HIV-infected mothers in Africa: a pooled analysis. Lancet. 2004;364(9441):1236-43.

9. Abrams EJ, Wiener J, Carter R, et al. Maternal health factors and early pediatric antiretroviral therapy influence the rate of perinatal HIV-1 disease progression in children. AIDS. 2003;17:867-77.

10. Motswere-Chirwa C, Voetsch A, Lu L, Letsholathebe V, Lekone P, Machakaire E, et al. Follow-up of infants diagnosed with HIV-early infant diagnosis program, Francistown, Botswana, 2005-2012. MMWR Morb Mortal Wkly Rep. 2014;63(7):158-60.

11. Violari A, Cotton MF, Gibb DM, et al. Early antiretroviral therapy and mortality among HIV-infected infants. N Engl J Med. 2008;359(21):2233-44.

12. Creek T, Tanuri A, Smith M, Seipone L, Smit M, et al. Early diagnosis of human immunodeficiency virus in infants using polymerase chain reaction on dried blood spots in Botswana's national program for prevention of mother-to-child transmission. Pediatr Infect Dis J. 2008;27(1):22-6.

13. Sherman GG, Stevens G, Jones SA, Horsfield P, Stevens WS. Dried blood spots improve access to HIV diagnosis and care for infants in low-resource settings. J Acquir Immune Defic Syndr. 2005;38(5):615-7.

14. Chatterjee A, Tripathi S, Gass R, Hamunime N, Panha S, Kiyaga C, et al. Implementing services for early infant diagnosis (EID) of HIV: a comparative descriptive analysis of national programs in four countries. BMC Public Health. 2011;11(1):553.

15. Diaz C, Hanson C, Cooper ER, et al. Disease progression in a cohort of infants with vertically acquired HIV infection observed from birth: the women and infants transmission study (WITS). J Acquir Immune Defic Syndr Hum Retrovirol. 1998;18(3):221-8.

16. Collins IJ, Jourdain G, et al. Long-term survival of HIV-infected children receiving antiretroviral therapy in Thailand: a 5-year observational cohort study. Clin Infect Dis. 2010;51(12):1449-57.

17. Chintu C, Bhat GJ, Walker AS, Mulenga V, Sinyinza F, Lishimpi H, Farrelly L, et al. Co-trimoxazole as prophylaxis against opportunistic infections in HIVinfected Zambian children (CHAP): a double-blind randomised placebocontrolled trial. Lancet. 2004;364(9448):1865-71.

18. Noubiap JJN, Bongoe A, Demanou SA. Mother-to-child transmission of HIV: findings from an early infant diagnosis program in Bertoua. Eastern Cameroon Pan Afr Med J. 2013;15:65.

19. Jick TD. Mixing qualitative and quantitative methods: triangulation in action. Adm Sci Q. 1979:602-11.

20. Uganda Ministry of Health. National Early Infant Diagnosis Training Manual. Uganda AIDS Control Programme. 2010. Kampala, Uganda.

21. Wendo C. HIV-positive mothers in Uganda resort to breastfeeding. Lancet. 2003;362(9383):542.

22. Kiyaga C, Sendagire H, Joseph E, McConnell I, Grosz J, Narayan V, et al. Uganda's new national laboratory sample transport system: a successful model for improving access to diagnostic services for early infant HIV diagnosis and other programs. PLoS One. 2013;8(11):e78609.

23. Kiyaga C, Sendagire H, Joseph E, Grosz J, McConnell I, Narayan V, et al. Consolidating HIV testing in a public health laboratory for efficient and sustainable early infant diagnosis (EID) in Uganda. J Public Health Policy. 2015;36(2):153-69.

24. Essajee S, Vojnov L, Penazzato M, Jani I, Siberry GK, Fiscus SA, Markby J. Reducing mortality in HIV-infected infants and achieving the 90-90-90 target through innovative diagnosis approaches. J Int AIDS Soc. 2015, 18(756). https://doi.org/10.7448/IAS.18.7.20299.

\section{Ready to submit your research? Choose BMC and benefit from}

- fast, convenient online submission

- thorough peer review by experienced researchers in your field

- rapid publication on acceptance

- support for research data, including large and complex data types

- gold Open Access which fosters wider collaboration and increased citations

- maximum visibility for your research: over $100 \mathrm{M}$ website views per year

At BMC, research is always in progress.

Learn more biomedcentral.com/submissions 